\title{
Of Microbes and Minds: A Narrative Review on the Second Brain Aging
}

\author{
Riccardo Calvani, Anna Picca*, Maria Rita Lo Monaco, Francesco Landi, \\ Roberto Bernabei and Emanuele Marzetti \\ Department of Geriatrics, Neurosciences and Orthopedics, Agostino Gemelli University Polyclinic, \\ Catholic University of the Sacred Heart, Rome, Italy
}

In recent years, an extensive body of literature focused on the gut-brain axis and the possible role played by the gut microbiota in modulating brain morphology and function from birth to old age. Gut microbiota has been proposed as a relevant player during the early phases of neurodevelopment, with possible long-standing effects in later life. The reduction in gut microbiota diversity has also become one of the hallmarks of aging, and disturbances in its composition are associated with several (age-related) neurological conditions, including depression, Alzheimer's disease, and Parkinson's disease. Several pathways have been evoked for gut microbiota-brain communication, including neural connections (vagus nerve), circulating mediators derived by host-bacteria cometabolism, as well as the influence exerted by gut microbiota on host gut function, metabolism, and immune system. Although the most provoking data emerged from animal studies and despite the huge debate around the possible epiphenomenal nature of those findings, the gut microbiota-brain axis still remains a fascinating target to be exploited to attenuate some of the most burdensome consequences of aging.

Keywords: gut microbiota, neurological disorders, inflamm-aging, gut-brain crosstalk, gut metabolism, brain development, Alzheimer, Parkinson

\section{GUT MICROBIOTA AND CENTRAL NERVOUS SYSTEM (CNS) IN HEALTH AND DISEASE: I "GUT" A FEELING}

Over the past decades, few aspects of human physiology have attracted the interest of researchers all over the world as the interaction between gut microbiota and human host (1). According to the current literature, the human holobiont (or superorganism) contains at least the same number of microorganisms (bacteria, archaea, fungi, and viruses) as its own cells (2). More than a billion years of mammalian-microbial coevolution have shaped a life-long interdependency (3). Growing evidence suggests that gut microbiota may be "at the intersection of everything," being implicated in virtually all physiological or pathological situations (1). Gut microbiota has been implicated in the maturation and modulation of the host immune response (4), interactions (positive and negative) with pathogens (5), regulation of bone density (6), vitamin biosynthesis (7), intestinal $5-10 \%$ of daily host energy requirements derives from gut microbiota metabolic activities (8).

Not surprisingly, gut microbiota composition and activities have been associated with a plethora of conditions, ranging from obesity to cardiovascular disease, chronic inflammatory diseases, and cancer (9-11).

Recently, a great emphasis has been placed on the role of intestinal microbiota in regulating the gut-brain axis (12-15). Gut microbiota and brain may influence one another through several pathways (Figure 1). Gut microbes-brain bidirectional communication is mediated by the vagus nerve that conveys information from the gastrointestinal tract to the CNS and back from CNS to 


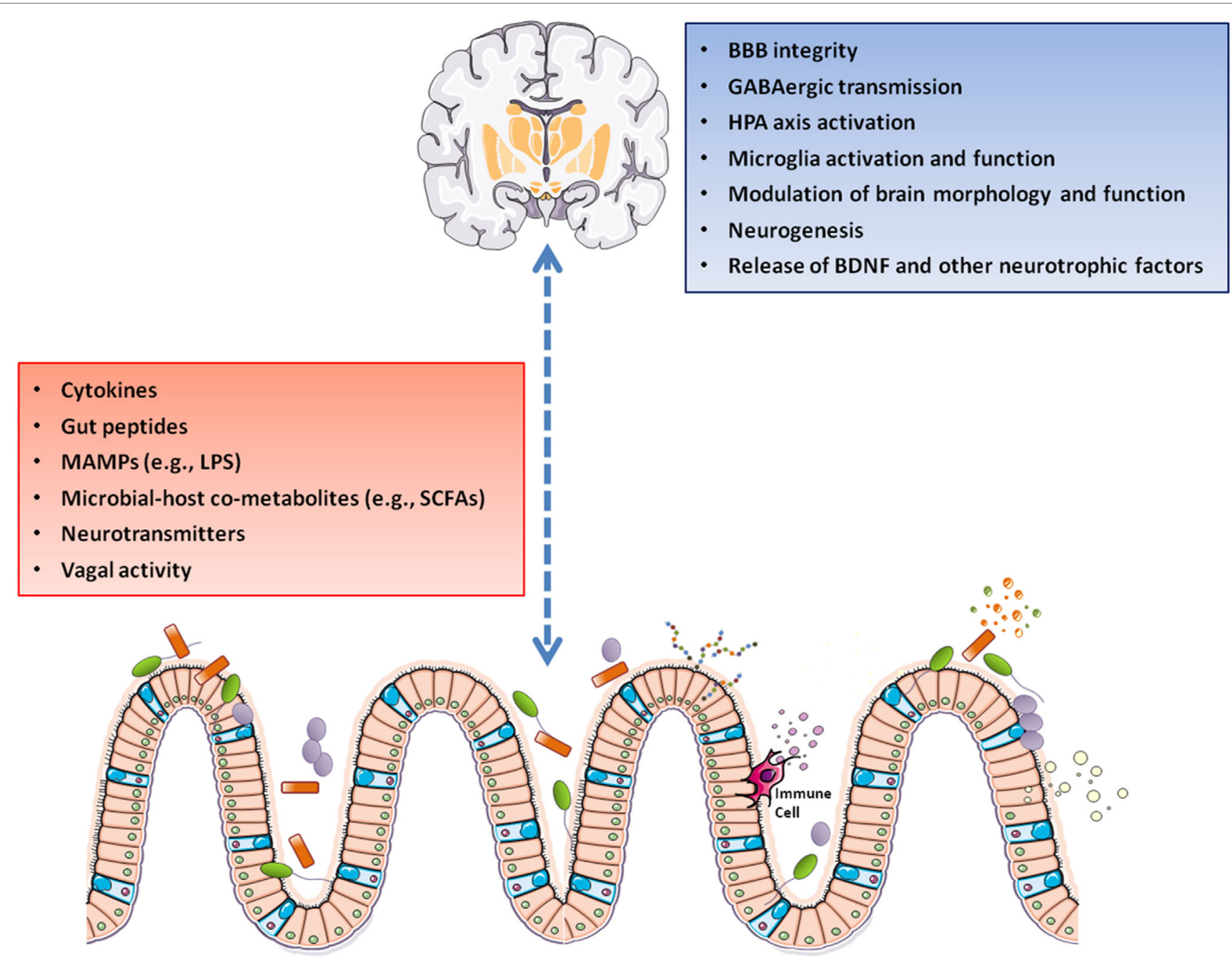

FIGURE 1 | A gut-brain axis supports the interactions between gut microbiota and the CNS through direct and indirect pathways involving vagal nerve activation, cytokine production, and release of neuropeptide/neurotransmitters and SCFAs. These mediators can pass the BBB and control the maturation and activation of brain immune cells (microglia). Following its activation, microglia modulates immune surveillance, synaptic pruning, and clearance of debris. On the other side, the HPA axis can suppress microglia activation, as well as influence cytokine release and trafficking of monocytes from the periphery to the brain. Abbreviations: BBB, blood-brain barrier; BDNF, brain-derived neurotrophic factor; GABA, gamma-aminobutyric acid; HPA axis, hypothalamic-pituitary-adrenal axis; LPS, lipopolysaccharide; MAMPs, microbe-associated molecular patterns; SCFAs, short-chain fatty acids; CNS, central nervous system.

the intestine to modulate intestinal motility, release of neurotransmitters and intestinal immune tone $(16,17)$. The sympathetic branch of the autonomic nervous system is also involved in intestinal homeostasis and gut immune regulation (18). Gut microbiota may also synthesize (or modulate the synthesis of) a number of neurotransmitters, including dopamine (DA), serotonin (5-HT), noradrenaline (NA), and gamma-aminobutyric acid (19-22). The hypothalamic-pituitary-adrenal axis (HPA axis) is another bidirectional route of communication through which host and gut microbes may interact to orchestrate the core response to both physical and psychological stress challenges (23-25). Bacterial metabolic activities may influence host metabolism and lead to the production of metabolites with neuroactive properties, including short-chain fatty acids (SCFAs) and dietary amino acid catabolites $(26,27)$. Finally, bacterial mediators in the forms of microbe-associated molecular patterns may drive neuroinflammation (28).

Through all these pathways, gut microbiota exerts a widespread influence on key neurological and behavioral processes and may be involved in critical phases of neurodevelopment and neurodegenerative disorders $(12-14,29)$. In this scenario, microbial activities on gut-brain axis seem to be especially relevant at the two extremities of human life course $(13,15)$. Early-life gut microbiota may play a role in shaping neuronal networks influencing cognitive, emotional, and social domains (13). Aging is associated with a reduction in microbial complexity, while alterations in intestinal microbiota composition, structure, and function have been retrieved in older individuals with Alzheimer's disease (AD) and Parkinson's disease (PD) $(30,31)$. 
In this narrative review, recent evidence on life-long gut microbiota-brain axis is summarized, with a particular focus on aging and age-related neurodegenerative diseases. All accessible relevant studies written in English were included.

\section{GUT MICROBIOTA AND NEURODEVELOPMENT: EARLY ORIGINS OF LATE NEUROLOGICAL DISEASES?}

The notion of "developmental origins of health and disease" poses that prenatal and perinatal life stages are critical periods in which environmental stimuli exert direct and indirect effects on the fetus that might be reflected in later health and disease conditions (32). In this context, early host-microbiota interactions seem to be among the most relevant factors in "programming" adult phenotypes (33). It has been postulated that a succession of microbiota components occurs through major steps at birth (depending on the timing and mode of delivery), then during breastfeeding and first interactions with the environment, and finally during and after weaning. Maternal-host factors (genetic background of mother-infant dyad) and perinatal exposure to antibiotics are among the most relevant factors in shaping the newborn's microbiota $(33,34)$. Interaction with colonizing microbiota may prime immune and metabolic functions and have a long-lasting influence on the risk of developing several conditions in later life, including gastrointestinal, allergic, autoimmune, and metabolic diseases (34).

Neurodevelopment is one of the most complex and fascinating aspects of human physiology that may be affected by early contact with gut microbiota $(12-14,35)$. Human brain development starts during the third gestational week and lasts through adolescence and into early adulthood in humans under the control of both genetic and environmental factors (36). The development of cognitive, emotional, and social brain circuits occur in parallel under the fine modulation by several molecular regulatory networks $(37,38)$. Critical windows in brain development have been described, during which neural circuits are particularly sensitive and even vulnerable to external factors, including gut microbiota composition $(39,40)$. Interestingly, early post-natal brain development overlap with gut microbiota establishment $(33,39,40)$.

Animal models, in particular germ-free (GF) mice, have been crucial for the study of gut microbiota-brain axis in early phases of neurodevelopment (41). Seminal studies suggest that both the composition and the metabolic activity of gut microbiota at specific time points may influence HPA axis development (42) and have long-lasting impact on behavioral and neuroendocrine responses to stress (42-45). Gut microbiota may program the activity of multiple neurotransmitter systems in different brain regions inducing a long-term modulation of motor control and anxiety-like behavior in adult life $(13,35,46)$. GF mice had a higher turnover rate of NA, DA, and serotonin 5-HT in the striatum compared with specific pathogen-free (SPF) mice (46). The serotonergic system seems to be particularly susceptible to early-life microbiota dynamics (47-50). Male GF animals showed a marked elevation in 5-HT and 5-hydroxyindoleacetic acid, its main metabolite, in the hippocampus compared with conventionally colonized control animals (48). Interestingly, post-weaning restoration of a normal flora failed to reverse the alterations in brain neurochemistry elicited by the lack of early life exposure to gut microbiota (48). Also, plasma 5-HT levels are affected by gut microbiota activity. In a metabolomics study, the colonization of GF mice induced a significant increase in plasma 5-HT (51), and bacterial metabolites were shown to stimulate 5 -HT synthesis and secretion by enterochromaffin cells (20,21). Intriguingly, the maternal separation in mice, an established model of early-life stress, induced profound changes in the gut microbiota that resulted in an anxiety-like phenotype (52).

The gut microbiota may also play a role in synapse maturation and synaptogenesis. In particular, GF animals when compared with SPF animals, showed higher striatal expression of synaptophysin and PSD 95, two markers of synaptogenesis and excitatory synapse maturation, respectively (46). Brain-derived neurotrophic factor (BDNF) is a key regulator of synaptic plasticity and neurogenesis in the brain and plays a crucial role in learning, memory, and mood regulation throughout life (53). In GF mice, Bdnf expression is significantly lower in the hippocampus, amygdala, and cingulate cortex compared with SPF mice (46). However, some inconsistency were reported about $B d n f$ expression in the hippocampus $(42,46,48,49)$.

Intriguingly, most of the reported neurodevelopmental alterations in GF mice occur differently in the two sexes (42, 46, $48,49)$. Gut microbiota influence on neurogenesis is relevant for the normal gross morphology and ultrastructure of the amygdala and hippocampus $(54,55)$. While GF mice exhibit increased adult hippocampal neurogenesis in the dorsal hippocampus, subsequent post-weaning microbial colonization failed to reverse these changes, suggesting the existence of a critical developmental window in early life during which gut microbiota may program adult hippocampal neurogenesis (55). Gut microbiota may also be instrumental for the development of the blood-brain barrier (BBB). GF mice, starting from intrauterine life, displayed a life-long increased BBB permeability compared with mice with a normal gut flora that can partially be reverted by the exposure to pathogen-free gut microbiota during adult life (56).

Microglia, the macrophages that constitute the first-line immune defense of the CNS, play a central role in brain development, plasticity, and cognition and have been associated with the initiation or progression of several developmental and neurodegenerative diseases, including $\mathrm{AD}$ and $\mathrm{PD}(57,58)$. Very recently, it was shown that microglia exhibited a timeand sex-specific susceptibility to gut microbiota depletion in mice (59). In particular, males seem to have their critical window during early in utero development, while females are more affected during adulthood. Microbiota alterations may have both acute and long-term effects on microglial functions. Remarkably, human fetal microglia showed significant similarities in the expression of key microglial genes when compared with murine counterparts (59). Finally, GF mice exhibited an increased myelination of neurons in the prefrontal cortex that could be reversed by colonization with a conventional microbiota following weaning (60). 
Interventions on the early gut microbiota community (through the use of antibiotics, drastic changes in diet and/or pre/probiotic administration) may have profound effects on the gut-brain axis throughout life. For instance, antibiotic use during the first years of life was associated with neurocognitive outcomes later in life (e.g., depression, behavioral difficulties) (61).

In summary, several lines of evidence, although obtained mostly from animal models, suggest a relevant role played by the gut microbiota during the early phases of neurodevelopment, with possible long-standing effects later in life. The translatability of animal model findings to humans is obviously a priority but, also when ascertained, a comprehensive discussion should be started before implementing intervention strategies that could harm the mother-infant dyad in the first critical 1,000 days of life $(62,63)$.

\section{THE ADULT "STEADY-STATE" MICROBIOTA AND CNS: COMMITTING TO A STABLE RELATIONSHIP}

From birth till adulthood, bacterial diversity and functional capacity expand progressively, although at different rates across life stages (i.e., faster during infancy and slightly slower in early childhood) $(64,65)$.

In adulthood, gut microbial population fluctuates around a steady state (in terms of composition, diversity, and function) and remains quite resilient unless gross perturbations occur (e.g., major health conditions) (66). "Healthy" adult gut microbiota are consistently dominated by 2 main phyla (Bacteroidetes and Firmicutes), but more than 1,000 different bacterial species have been characterized and represent the vast human microbial collection (67-69). Each individual is characterized by a specific combination and proportion of different microbial species and subspecies (strains) that constitutes a unique microbial fingerprint (69). Despite this taxonomic inter-individual variability, adult gut microbiota display a relatively consistent functional capacity in healthy persons $(70,71)$. Importantly, microbial diversity and functional redundancy are positively associated with health, while decreased microbial richness and diversity and loss of functional redundancy characterize the microbiota in multiple disease conditions $(66,69,72)$. Adult gut microbiota is influenced by several factors, including host genetics (73), nutrition and dietary habits $(74,75)$, xenobiotics (e.g., antibiotics) and other drugs (76-78), exercise $(75,79,80)$, and circadian rhythm $(81,82)$.

Gut microbiota and brain dynamically interact also during adulthood. In adult mice, short-term oral administration of broad-spectrum antibiotics induced a decrease in anxiety and upregulated hippocampal expression of $B d n f(83)$. These changes were associated with a transient perturbation of microbiota but occurred independent of inflammatory status, vagal or sympathetic integrity, or alterations in gastrointestinal neurotransmitter levels (83). Adult neuroplasticity is sensitive to several environmental stimuli, including stress and gut microbiota alterations (84). Adult mice treated with antibiotics showed decreased hippocampal neurogenesis and memory retention (85). This effect was not completely rescued by the restoration of a normal flora by fecal transplant, unless supported by exercise or a probiotic cocktail administration (85).

Recent evidence suggests that complex microbiota-derived stimuli are requested for microglia maintenance also during adulthood $(26,59,86)$. In particular, SCFAs, derived from bacterial fermentation processes, seem to regulate adult microglia homeostasis (26). Moreover, short-term antibiotic treatment in adult mice induce a rapid and sexually dimorphic (higher in females) change in microglial gene expression, reinforcing the concept that microbiota perturbations may have a relevant impact of microglia also during adulthood (59).

\section{THE SECOND BRAIN AGING: LINKING GUT MICROBIOTA TO NEURODEGENERATION}

Aging is a process characterized by progressive functional decline of all physiological systems. In the gastrointestinal tract, aging involves the degeneration of enteric nervous system (ENS), alterations in gastrointestinal motility, perturbations in small intestinal permeability and mucosal defense system, which may promote the development of gastrointestinal diseases, affect the local and systemic inflammatory status, and deeply influence both the composition and function of resident microbiota (87-89).

Aging is also associated with broad changes in brain and whole body physiology that may influence gut microbiota-brain axis. In particular, the HPA axis is deeply perturbed, through a self-reinforcing cycle mediated by the hyperactivation of the HPA axis that leads to increased basal glucocorticoid release and the impaired HPA negative feedback due to reduced central glucocorticoid receptor expression $(90,91)$. HPA axis dysfunctions have been associated with decline in hippocampal volume and cognitive performance, and increased risk of late-life depression and anxiety $(92,93)$. Also circadian rhythm disruption, which is typical of aging, may be involved in this process, due to the potential effect on both cortisol level fluctuations and gut microbial activities $(94,95)$.

The aging brain is also deficient in the synthesis of neurotrophic factors, including BDNF (96) as well as several neurotransmitters, including 5-HT and DA, all of which lead to neuronal and cognitive dysfunction $(97,98)$. BBB breakdown is an early event in the aging human brain that begins in the hippocampus and may contribute to cognitive impairment (99).

Aging is also characterized by the progressive decline in immune function (immunosenescence) associated with a chronic, low-grade inflammation (inflamm-aging) $(100,101)$. Both processes may have many effects on the CNS, such as microglial activation, BBB breakdown, and increase in oxidative damage that may contribute to neurodegenerative and neuropsychiatric diseases (100). Remarkably, recent data suggest that, in old mice, gut microbiota contribute to inflamm-aging, and that this inflammatory phenotype may be transferred to young GF mice (102).

Major taxonomic shifts and a consistent decrease in microbial richness and diversity have been reported in people 65 years of age and older and these changes were associated with worsening 
of health status and frailty $(89,103)$. Similar findings were also obtained in mice (104).

The characterization of gut microbiota of centenarians revealed the presence of significant compositional differences across life stages till extreme ages (105). In particular, core microbiota (mostly composed by the members of Ruminococcaceae, Lachnospiraceae, and Bacteroidaceae families) seem to accompany human life, decreasing in abundance along with aging (105). In longevity and extreme longevity, an enrichment in some subdominant health-associated groups (e.g., Akkermansia, Bifidobacterium, and Christensenellaceae) occurs, even with the support of some opportunistic and allochthonous bacteria (105).

Recently, the effects of aging on the microbiota gut-brain axis were assessed in male mice (106). Aged mice showed significant shifts in gut microbiota that were associated with deficits in spatial memory and increases in anxiety-like behaviors compared with young adult mice (106). These changes were positively correlated with the abundance of bacteria from the Porphyromonadaceae family. Aged mice also exhibited increased gut permeability that was associated with elevations in peripheral pro-inflammatory cytokines (106).

These preliminary findings suggest that age-related changes in gut microbiota may impact behavioral and cognitive functions and support the relevance of the alteration in gut permeability and peripheral inflammation in mediating these effects.

As outlined earlier, the possible link between early gut microbiota-brain interactions and late onset neurological conditions, including $\mathrm{AD}$ and $\mathrm{PD}$, is an intriguing area of research (15).

\section{Alzheimer's Disease}

In $\mathrm{AD}$, the most common form of age-related dementia, deposition of protein aggregates composed of amyloid- $\beta$ (A $\beta)$ peptide and tau in brain tissues impairs cognitive function (107). Both host- and environmental factors that regulate these processes have been described, including a potential role for gut microbiota (108) (Figure 2).

In $\mathrm{AD}$, reduced microbial richness and diversity were observed, with low abundance of Firmicutes and Bifidobacterium and increased Bacteroidetes that characterized the microbiome of $\mathrm{AD}$ patients (108). Correlations were found between the levels of Bacteroides, Turicibacter, and SMB53 and the concentration of glial activation biomarkers in cerebrospinal fluid of AD (108).

An increase in the abundance of the pro-inflammatory Escherichia/Shigella taxon, and a corresponding reduction in the anti-inflammatory E. rectale was associated with higher levels of inflammatory mediators in patients with cognitive impairment and brain amyloidosis (109). Also in a mouse model overexpressing amyloid precursor protein and presenilin 1 (APPPS1), a distinct microbial signature was observed with an increase in Rikenellaceae and decreased Allobaculum and Akkermansia compared with age-matched wild-type controls (110).

Interestingly, reduced levels of Akkermansia characterize gut microbiota of mice with obesity and type 2 diabetes (111), two potentially modifiable risk factors for AD (107). Importantly, both young and old GF APPPS1 transgenic mice displayed a drastic reduction of cerebral $A \beta$ pathology when compared with control mice, along with a reduced microgliosis (110). Further to

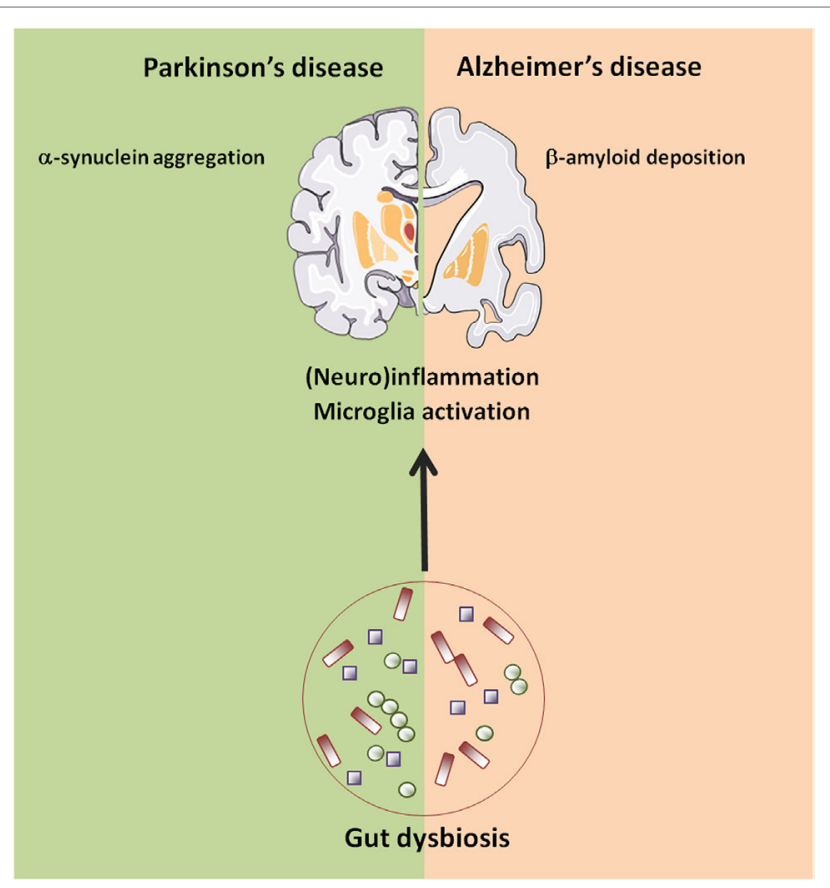

FIGURE 2 | Age-related changes in gut-brain axis possibly involved in neurodegeneration. Abbreviations: AD, Alzheimer's disease; PD, Parkinson's disease.

this, colonization of GF-APPPS1 transgenic mice with microbiota from conventionally raised APPPS 1 transgenic mice increased cerebral A $\beta$ pathology, while colonization with microbiota from wild-type mice was less effective in increasing cerebral $A \beta$ levels (110). Notably, GF-APPPS1 displayed increased levels of the $\mathrm{A} \beta$-degrading enzymes insulin degrading enzyme and neprilysin degrading enzyme, suggesting a mechanism through which gut microbiota influence cerebral A $\beta$ amyloidosis (110).

In the same mouse model of $\mathrm{AD}$, life-long antibiotic treatment induced a considerable perturbation in gut microbial composition (including an expansion of Akkermansia) that was associated with marked changes in the circulating cytokine/ chemokine network, a striking reduction in amyloid plaque deposition, and a concomitant increase in soluble $\mathrm{A} \beta$ (112). This was accompanied by alterations in neuroinflammatory milieu that lead to reduced plaque-localized gliosis and altered microglial morphology (112). Remarkably, early post-natal antibiotic treatment alone resulted in long-term alterations in gut microbial genera that were associated with changes in the inflammatory environment of serum and cerebrospinal fluid and attenuated $\mathrm{A} \beta$ amyloidosis in a manner similar to that observed in mice subjected to life-long antibiotic selection pressure (113). These findings corroborate the hypothesis of the presence of critical developmental periods in which the commensal microbiota manipulation may have long-lasting effects on host immunity and potential implications for neurodegenerative diseases.

In another model of $\mathrm{AD}$, the $5 \mathrm{xFAD}$ transgenic mouse, elevated levels of APP were found not only in the brain but also in the different gut districts and this was associated with a distinct fecal 
microbiota profile relative to wild-type animals, with an increase in pro-inflammatory species (e.g., Clostridium leptum) (114).

Alterations in gut microbiota composition together with the increase in intestinal permeability with age may lead to the translocation of microbes or microbial components [i.e., lipopolysaccharide (LPS)] from the gut to induce systemic and CNS inflammation (115). Interestingly, in vitro and in vivo studies have demonstrated a possible association between LPS and AD pathology. Coincubation of A $\beta$ peptide with LPS potentiated amyloid fibril formation (116), and systemic administration of LPS in wild-type and transgenic AD mice induced neuroinflammation, amyloid deposition, and tau pathology (117-119). Moreover, in postmortem brain parenchyma and blood vessels from patients with $\mathrm{AD}$, levels of LPS and Gram-negative E. coli fragments were greater compared with control brains and colocalized with amyloid plaques (120).

While the study of the microbiota gut-brain axis in $\mathrm{AD}$ is still in its infancy, promising preclinical data suggest that the modulation of gut microbiota through dietary ingredients or probiotics may provide a means to counteract the development or progression of neurodegenerative disease. For instance, in a triple-transgenic mouse model of $\mathrm{AD}(3 \mathrm{xTg}-\mathrm{AD})$, a formulation of lactic acid bacteria and bifidobacteria changed the composition of gut microbiota, stimulated the production of beneficial metabolites (e.g., increased SCFAs), reduced the levels of proinflammatory cytokines, increased gut hormones concentration and positively modulate quality control processes and proteolysis, reducing $A \beta$ load and improving cognitive function (121). Moreover, the administration of the probiotic mixture VSL\#3 to aged rats induced a robust perturbation in gut microbiota composition, that was accompanied by gene expression changes in the brain cortex, attenuated age-related deficits in long-term potentiation, decreased microglial activation, and increased BDNF and synapsin levels (122). In addition, 3-hydroxybenzoic acid and 3-(3-hydroxyphenyl)propionic acid, the phenolic products of microbial conversion of grape seed polyphenol extracts (and other dietary polyphenols), may potently interfere with the assembly of $A \beta$ peptides into neurotoxic $A \beta$ aggregates in vitro (123).

Despite these interesting preliminary findings, more work is needed to determine whether gut microbiota modulation may be employed for the prevention and/or treatment of $\mathrm{AD}$ pathogenic processes.

\section{Parkinson's Disease}

Parkinson's disease is the second most common neurodegenerative disorder, affecting $2-3 \%$ of the population $\geq 65$ years of age $(124,125)$. Degeneration of the dopaminergic nigro-striatal pathway and widespread intracellular $\alpha$-synuclein accumulation are the neuropathological hallmarks of PD that are associated with bradykinesia and other cardinal motor and non-motor features (126).

Gastrointestinal dysfunction, in particular in the form of constipation, is among the most frequent prodromal nonmotor symptoms of PD that may precede motor symptoms by decades (126). At later disease stages, oral issues including drooling and swallowing problems and delays in gastric emptying further exacerbate gastrointestinal dysfunction (127). Aggregates of $\alpha$-synuclein have been retrieved in the mucosal and submucosal nerve fibers and ganglia of the ENSs of PD patients at early disease stages $(128,129)$. In addition, some observations from experimental models support the intriguing hypothesis that intestinal $\alpha$-synuclein may spread to the brain via postganglionic enteric neurons and the vagus nerve (130). Interestingly, the risk of developing PD was significantly decreased in patients who underwent a full truncal vagotomy compared with those who underwent selective vagotomy and in the general population (131).

Not surprisingly, gastrointestinal disturbances in people with PD are accompanied by alterations in fecal and mucosal microbial populations $(31,132-134)$. In particular, a reduced abundance of Prevotellaceae, mucin producers that regulate intestinal permeability, was commonly reported in PD patients $(31,132,135,136)$, while Enterobacteriaceae were positively associated with the severity of postural instability and gait difficulty (31). Clostridium coccoides group was high in early PD patients, while Lactobacillus gasseri subgroup was high in advanced PD patients (132). A pro-inflammatory dysbiosis, characterized by low counts of "anti-inflammatory" butyrate-producing bacteria from the genera Blautia, Coprococcus, and Roseburia and higher "pro-inflammatory" Proteobacteria of the genus Ralstonia was also reported in individual with PD (133). Individuals affected by PD also showed lower levels of SCFA concentrations, derived from host-microbiota cometabolism, that may have neuroactive and immunomodulating properties (135). Other evidence of microbiota dysregulation in PD includes small intestine bacterial overgrowth and high rates of Helicobacter pylori infection (137, 138). It is worth noting that this infection has also been involved in the pathogenesis of AD (139). Finally, the total abundance of intestinal bacterial was found to decrease during PD progression, with a low count of Bifidobacterium associated with worsening of PD symptoms (134).

Collectively, these findings suggest that perturbations in gut microbiota structure and function may be associated with the development and progression of PD through several potential mechanisms, including inflammation and bacterial translocation (Figure 2). However, findings in humans remain largely descriptive. Again, animal models provided some useful insights into the physiopathological mechanisms linking gut dysbiosis to PD. Under GF conditions, or when bacteria were depleted in post-natal life following antibiotic treatment, transgenic mice overexpressing $\alpha$-synuclein showed reduced microglia activation, $\alpha$-synuclein inclusions, gastrointestinal symptoms, and motor deficits compared with animals with a complex microbiota (140). Moreover, administration of a mixture of microbially derived SCFAs (acetate, propionate, and butyrate) restored all major features of PD in GF mice, suggesting that microbial metabolic mediators may promote microglia activation and $\alpha$-synuclein aggregation and contribute to motor dysfunction in PD (140). Remarkably, mice transplanted with PD microbiota compared with mice who received microbiota from healthy human controls displayed enhanced motor dysfunction, suggesting that dysbiosis may be the environmental factor that combined with a genetic 


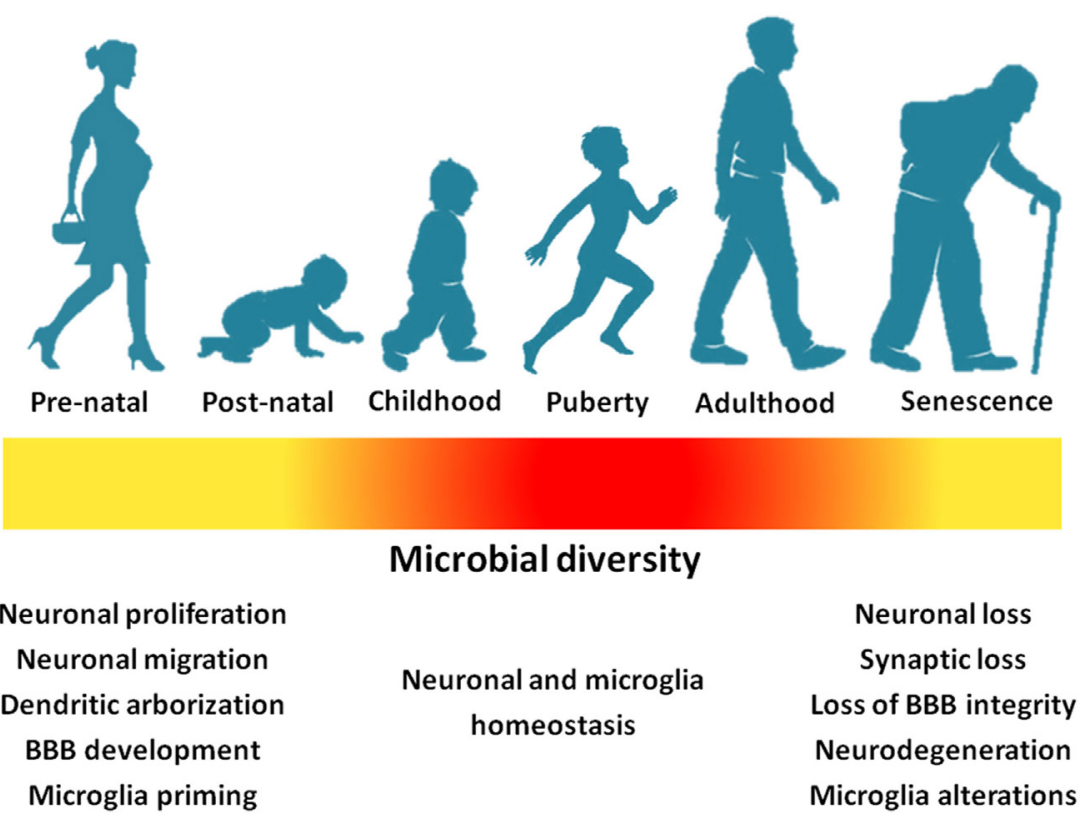

FIGURE 3 | Gut microbial richness and diversity across life stages impact neurodevelopment and the central nervous homeostasis (yellow: low richness/diversity; red: high richness/diversity). Abbreviation: BBB, blood-brain barrier.

predisposition ( $\alpha$-synuclein overexpression) influences disease outcomes in mice (140).

As already outlined for $\mathrm{AD}$, in neurodegenerative diseases, including PD, the passage of bacterial products from the intestine to the circulation and into brain, or "molecular mimicry" processes induced by bacterial amyloids may trigger a persistent neuroinflammation $(28,141,142)$ that in turn contributes to neuronal dysfunction and death (143). In this scenario, it has recently been proposed that $A \beta$ production and aggregation may originally act as an antimicrobial defense and then infectious or sterile inflammatory stimuli may drive amyloidosis (144).

While it is currently recommended the use of fermented milk containing probiotics and prebiotic fiber in PD patients with constipation (145), the possible beneficial effects of the manipulation of gut microbiota (through diet, live bacteria, or microbiota transplantation) on the initiation or progression of the neurodegenerative process have not yet been explored. Further studies are also needed to assess the possible interactions among these interventions and levodopa uptake and availability.

\section{CONCLUDING REMARKS}

At the beginning of the twentieth century, the Nobel Prize winner Elie Metchnikoff theorized in his tracts, The Nature of Man: Studies in Optimistic Philosophy (1903) and The Prolongation of Life: Optimistic Studies (1907), that health status could be improved and senility delayed by replacing the native gut microbes with lactic acid bacteria such as those present in yogurt (146). In the past few decades, this idea was resumed and updated under the influence of methodological and technological advances in science (147). A more ecological perspective was then embraced and the concepts of complexity, (dis)harmony, (Nash)equilibrium, and personalization/precision were introduced to capture the dynamic aspects of gut microbiota-host relationship (66, 147-149).

While the study of microbiota gut-brain axis is still in infancy, a number of potential mechanisms (and, hence, plausible targets) have begun to be unveiled. Early-life interactions between host and colonizing gut microbes seem to influence the way in which the nervous system starts obtaining information about the external and internal environment in critical phases of neurodevelopment. BBB establishment and function, central inflammatory processes and neurogenesis may be differentially affected by the gut microbial assemblies and their metabolic products (Figure 3). Evidence is also accumulating for a role of life-long microbiota-host interactions in age-related disorders such as $\mathrm{AD}$ and $\mathrm{PD}$.

Taken together, these data open up the possibility of developing interventions targeting the gut microbiota (in particular at the extreme ages of life) to improve brain health. Preclinical studies have suggested the efficacy of the modulation of the gut microbiota in ameliorating conditions such as depression and neurodegenerative diseases (150). A new term, "psychobiotics" (and related "psychobiotic properties"), was coined to define live bacteria (probiotics) and nutritional support for such bacteria (prebiotics), but also virtually any exogenous factor, such as diet, exercise, and drugs, acting on brain through bacterially mediated effects (19).

Despite the "optimistic nature" of this 100-year-old idea, future research should tackle several challenging questions before truly effective interventions in humans may be implemented. For instance, most of the published studies have only associated the gut 
microbiota with diseases without proving any causation $(1,151)$. It is therefore crucial to assess whether changes in microbiota underpin disease pathophysiology or are just epiphenomena. Further to this, the microbial properties that are necessary to support proper neurodevelopment and prevent neurodegeneration should be clearly established. In addition, sufficiently powered, rigorous clinical trials should be conducted to assess the translatability of animal model findings to human conditions.

\section{AUTHOR CONTRIBUTIONS}

RC, EM, and AP conceived the manuscript. RC, MRLM, and AP drafted the paper. RB, FL, and EM supervised and edited the

\section{REFERENCES}

1. Cani PD. Gut microbiota-at the intersection of everything? Nat Rev Gastroenterol Hepatol (2017) 14:321-2. doi:10.1038/nrgastro.2017.54

2. Sender R, Fuchs S, Milo R. Are we really vastly outnumbered? Revisiting the ratio of bacterial to host cells in humans. Cell (2016) 164:337-40. doi:10.1016/j.cell.2016.01.013

3. Lynch SV, Pedersen O. The human intestinal microbiome in health and disease. N Engl J Med (2016) 375:2369-79. doi:10.1056/NEJMra1600266

4. Postler TS, Ghosh S. Understanding the holobiont: how microbial metabolites affect human health and shape the immune system. Cell Metab (2017) 26:110-30. doi:10.1016/j.cmet.2017.05.008

5. Bäumler AJ, Sperandio V. Interactions between the microbiota and pathogenic bacteria in the gut. Nature (2016) 535:85-93. doi:10.1038/ nature 18849

6. Jones RM, Mulle JG, Pacifici R. Osteomicrobiology: the influence of gut microbiota on bone in health and disease. Bone (2017). doi:10.1016/j.bone. 2017.04.009

7. LeBlanc JG, Milani C, de Giori GS, Sesma F, van Sinderen D, Ventura M. Bacteria as vitamin suppliers to their host: a gut microbiota perspective. Curr Opin Biotechnol (2013) 24:160-8. doi:10.1016/j.copbio.2012.08.005

8. Macfarlane GT, Gibson GR. Carbohydrate fermentation, energy transduction and gas metabolism in the human large intestine. In: Mackie RL, White BA, editors. Gastrointestinal Microbiology. Boston, MA: Springer (1997). p. 269-318.

9. Torres-Fuentes C, Schellekens H, Dinan TG, Cryan JF. The microbiota-gut-brain axis in obesity. Lancet Gastroenterol Hepatol (2017) 2:747-56. doi:10.1016/S2468-1253(17)30147-4

10. Marques FZ, Mackay CR, Kaye DM. Beyond gut feelings: how the gut microbiota regulates blood pressure. Nat Rev Cardiol (2018) 15:20-32. doi:10.1038/ nrcardio. 2017.120

11. Garrett WS. Cancer and the microbiota. Science (2015) 348:80-6. doi:10.1126/ science.aaa4972

12. Sharon G, Sampson TR, Geschwind DH, Mazmanian SK. The central nervous system and the gut microbiome. Cell (2016) 167:915-32. doi:10.1016/j. cell.2016.10.027

13. Kelly JR, Minuto C, Cryan JF, Clarke G, Dinan TG. Cross talk: the microbiota and neurodevelopmental disorders. Front Neurosci (2017) 11:490. doi:10.3389/fnins.2017.00490

14. Dinan TG, Cryan JF. Gut instincts: microbiota as a key regulator of brain development, ageing and neurodegeneration. J Physiol (2017) 595:489-503. doi: $10.1113 /$ JP273106

15. Sherwin E, Dinan TG, Cryan JF. Recent developments in understanding the role of the gut microbiota in brain health and disease. Ann N Y Acad Sci (2017). doi:10.1111/nyas.13416

16. Forsythe P, Bienenstock J, Kunze WA. Vagal pathways for microbiome-brain-gut axis communication. Adv Exp Med Biol (2014) 817:115-33. doi:10.1007/978-1-4939-0897-4_5

17. Andersson U, Tracey KJ. Reflex principles of immunological homeostasis. Annu Rev Immunol (2012) 30:313-35. doi:10.1146/annurev-immunol-020711075015 manuscript. All authors read and approved the final version of the paper.

\section{ACKNOWLEDGMENTS}

This study was supported by the "Centro Studi Achille e Linda Lorenzon" (AP, EM, and RC), intramural research grants from the Catholic University of the Sacred Heart (D3.2 2013 and D3.2 2015, FL), Innovative Medicines Initiative Joint Undertaking (IMI-JU 115621, EM, FL, RB, and RC), and Fondazione Roma (NCDs Call for Proposal 2013, AP and RC). The figures were drawn using the freely available Servier Medical Art resource (http://www.servier.com/Powerpoint-image-bank).

18. de Jonge WJ. The gut's little brain in control of intestinal immunity. ISRN Gastroenterol (2013) 2013:630159. doi:10.1155/2013/630159

19. Sarkar A, Lehto SM, Harty S, Dinan TG, Cryan JF, Burnet PWJ. Psychobiotics and the manipulation of bacteria-gut-brain signals. Trends Neurosci (2016) 39:763-81. doi:10.1016/j.tins.2016.09.002

20. Reigstad CS, Salmonson CE, Rainey JF, Szurszewski JH, Linden DR, Sonnenburg JL, et al. Gut microbes promote colonic serotonin production through an effect of short-chain fatty acids on enterochromaffin cells. FASEB $J(2015)$ 29:1395-403. doi:10.1096/fj.14-259598

21. Yano JM, Yu K, Donaldson GP, Shastri GG, Ann P, Ma L, et al. Indigenous bacteria from the gut microbiota regulate host serotonin biosynthesis. Cell (2015) 161:264-76. doi:10.1016/j.cell.2015.02.047

22. Barrett E, Ross RP, O’Toole PW, Fitzgerald GF, Stanton C. $\gamma$-Aminobutyric acid production by culturable bacteria from the human intestine. J Appl Microbiol (2012) 113:411-7. doi:10.1111/j.1365-2672.2012.05344.x

23. Foster JA, Rinaman L, Cryan JF. Stress \& the gut-brain axis: regulation by the microbiome. Neurobiol Stress (2017) 7:124-36. doi:10.1016/j. ynstr.2017.03.001

24. Mudd AT, Berding K, Wang M, Donovan SM, Dilger RN. Serum cortisol mediates the relationship between fecal Ruminococcus and brain $\mathrm{N}$-acetylaspartate in the young pig. Gut Microbes (2017) 8:589-600. doi:10.1080/19490976.2017.1353849

25. Bravo JA, Forsythe P, Chew MV, Escaravage E, Savignac HM, Dinan TG, et al. Ingestion of Lactobacillus strain regulates emotional behavior and central GABA receptor expression in a mouse via the vagus nerve. Proc Natl Acad Sci U S A (2011) 108:16050-5. doi:10.1073/pnas.1102999108

26. Erny D, Hrabě de Angelis AL, Jaitin D, Wieghofer P, Staszewski O, David E, et al. Host microbiota constantly control maturation and function of microglia in the CNS. Nat Neurosci (2015) 18:965-77. doi:10.1038/nn.4030

27. Rothhammer V, Mascanfroni ID, Bunse L, Takenaka MC, Kenison JE, Mayo L, et al. Type I interferons and microbial metabolites of tryptophan modulate astrocyte activity and central nervous system inflammation via the aryl hydrocarbon receptor. Nat Med (2016) 22:586-97. doi:10.1038/nm.4106

28. Pal GD, Shaikh M, Forsyth CB, Ouyang B, Keshavarzian A, Shannon KM. Abnormal lipopolysaccharide binding protein as marker of gastrointestinal inflammation in Parkinson disease. Front Neurosci (2015) 9:306. doi:10.3389/ fnins.2015.00306

29. Fung TC, Olson CA, Hsiao EY. Interactions between the microbiota, immune and nervous systems in health and disease. Nat Neurosci (2017) 20:145-55. doi:10.1038/nn.4476

30. Jiang C, Li G, Huang P, Liu Z, Zhao B. The gut microbiota and Alzheimer's disease. J Alzheimers Dis (2017) 58:1-15. doi:10.3233/JAD-161141

31. Scheperjans F, Aho V, Pereira PAB, Koskinen K, Paulin L, Pekkonen E, et al. Gut microbiota are related to Parkinson's disease and clinical phenotype. Mov Disord (2015) 30:350-8. doi:10.1002/mds.26069

32. Gluckman PD, Hanson MA, Mitchell MD. Developmental origins of health and disease: reducing the burden of chronic disease in the next generation. Genome Med (2010) 2:14. doi:10.1186/gm135

33. Rautava S, Luoto R, Salminen S, Isolauri E. Microbial contact during pregnancy, intestinal colonization and human disease. Nat Rev Gastroenterol Hepatol (2012) 9:565-76. doi:10.1038/nrgastro.2012.144 
34. Goulet O. Potential role of the intestinal microbiota in programming health and disease. Nutr Rev (2015) 73(Suppl 1):32-40. doi:10.1093/nutrit/nuv039

35. Tognini P. Gut microbiota: a potential regulator of neurodevelopment. Front Cell Neurosci (2017) 11:25. doi:10.3389/fncel.2017.00025

36. Stiles J, Jernigan TL. The basics of brain development. Neuropsychol Rev (2010) 20:327-48. doi:10.1007/s11065-010-9148-4

37. Bassett DS, Sporns O. Network neuroscience. Nat Neurosci (2017) 20:353-64. doi:10.1038/nn.4502

38. Chai LR, Khambhati AN, Ciric R, Moore TM, Gur RC, Gur RE, et al. Evolution of brain network dynamics in neurodevelopment. Netw Neurosci (2017) 1:14-30. doi:10.1162/NETN_a_00001

39. Borre YE, O'Keeffe GW, Clarke G, Stanton C, Dinan TG, Cryan JF. Microbiota and neurodevelopmental windows: implications for brain disorders. Trends Mol Med (2014) 20:509-18. doi:10.1016/j.molmed.2014.05.002

40. Chu DM, Ma J, Prince AL, Antony KM, Seferovic MD, Aagaard KM. Maturation of the infant microbiome community structure and function across multiple body sites and in relation to mode of delivery. Nat Med (2017) 23:314-26. doi:10.1038/nm.4272

41. Luczynski P, McVey Neufeld K-A, Oriach CS, Clarke G, Dinan TG, Cryan JF. Growing up in a bubble: using germ-free animals to assess the influence of the gut microbiota on brain and behavior. Int J Neuropsychopharmacol (2016) 19:yw020. doi:10.1093/ijnp/pyw020

42. Sudo N, Chida Y, Aiba Y, Sonoda J, Oyama N, Yu X-N, et al. Postnatal microbial colonization programs the hypothalamic-pituitary-adrenal system for stress response in mice. J Physiol (2004) 558:263-75. doi:10.1113/ jphysiol.2004.063388

43. Nishino R, Mikami K, Takahashi H, Tomonaga S, Furuse M, Hiramoto T, et al. Commensal microbiota modulate murine behaviors in a strictly contamination-free environment confirmed by culture-based methods. Neurogastroenterol Motil (2013) 25:521-8. doi:10.1111/nmo.12110

44. Gacias M, Gaspari S, Santos P-MG, Tamburini S, Andrade M, Zhang F, et al. Microbiota-driven transcriptional changes in prefrontal cortex override genetic differences in social behavior. Elife (2016) 5:e13442. doi:10.7554/ eLife.13442

45. Moloney RD, Desbonnet L, Clarke G, Dinan TG, Cryan JF. The microbiome: stress, health and disease. Mamm Genome (2014) 25:49-74. doi:10.1007/ s00335-013-9488-5

46. Diaz Heijtz R, Wang S, Anuar F, Qian Y, Björkholm B, Samuelsson A, et al. Normal gut microbiota modulates brain development and behavior. Proc Natl Acad Sci U S A (2011) 108:3047-52. doi:10.1073/pnas.1010529108

47. O'Mahony SM, Clarke G, Borre YE, Dinan TG, Cryan JF. Serotonin, tryptophan metabolism and the brain-gut-microbiome axis. Behav Brain Res (2015) 277:32-48. doi:10.1016/j.bbr.2014.07.027

48. Clarke G, Grenham S, Scully P, Fitzgerald P, Moloney RD, Shanahan F, et al. The microbiome-gut-brain axis during early life regulates the hippocampal serotonergic system in a sex-dependent manner. Mol Psychiatry (2013) 18:666-73. doi:10.1038/mp.2012.77

49. Neufeld KM, Kang N, Bienenstock J, Foster JA. Reduced anxiety-like behavior and central neurochemical change in germ-free mice. Neurogastroenterol Motil (2011) 23(255-64):e119. doi:10.1111/j.1365-2982.2010.01620.x

50. El Aidy S, Kunze W, Bienenstock J, Kleerebezem M. The microbiota and the gut-brain axis: insights from the temporal and spatial mucosal alterations during colonisation of the germfree mouse intestine. Benef Microbes (2012) 3:251-9. doi:10.3920/BM2012.0042

51. Wikoff WR, Anfora AT, Liu J, Schultz PG, Lesley SA, Peters EC, et al. Metabolomics analysis reveals large effects of gut microflora on mammalian blood metabolites. Proc Natl Acad Sci U S A (2009) 106:3698-703. doi:10.1073/ pnas. 0812874106

52. De Palma G, Blennerhassett P, Lu J, Deng Y, Park AJ, Green W, et al. Microbiota and host determinants of behavioural phenotype in maternally separated mice. Nat Commun (2015) 6:7735. doi:10.1038/ncomms8735

53. Zuccato C, Cattaneo E. Brain-derived neurotrophic factor in neurodegenerative diseases. Nat Rev Neurol (2009) 5:311-22. doi:10.1038/nrneurol. 2009.54

54. Luczynski P, Whelan SO, O'Sullivan C, Clarke G, Shanahan F, Dinan TG, et al. Adult microbiota-deficient mice have distinct dendritic morphological changes: differential effects in the amygdala and hippocampus. Eur J Neurosci (2016) 44:2654-66. doi:10.1111/ejn.13291
55. Ogbonnaya ES, Clarke G, Shanahan F, Dinan TG, Cryan JF, O'Leary OF. Adult hippocampal neurogenesis is regulated by the microbiome. Biol Psychiatry (2015) 78:e7-9. doi:10.1016/j.biopsych.2014.12.023

56. Braniste V, Al-Asmakh M, Kowal C, Anuar F, Abbaspour A, Tóth M, et al. The gut microbiota influences blood-brain barrier permeability in mice. Sci Transl Med (2014) 6:263ra158. doi:10.1126/scitranslmed.3009759

57. Tay TL, Savage JC, Hui CW, Bisht K, Tremblay M-Ë. Microglia across the lifespan: from origin to function in brain development, plasticity and cognition. J Physiol (2017) 595:1929-45. doi:10.1113/JP272134

58. Colonna M, Butovsky O. Microglia function in the central nervous system during health and neurodegeneration. Annu Rev Immunol (2017) 35:441-68. doi:10.1146/annurev-immunol-051116-052358

59. Thion MS, Low D, Silvin A, Chen J, Grisel P, Schulte-Schrepping J, et al. Microbiome influences prenatal and adult microglia in a sex-specific manner. Cell (2017) 172:500-16. doi:10.1016/j.cell.2017.11.042

60. Hoban AE, Stilling RM, Ryan FJ, Shanahan F, Dinan TG, Claesson MJ, et al. Regulation of prefrontal cortex myelination by the microbiota. Transl Psychiatry (2016) 6:e774. doi:10.1038/tp.2016.42

61. Slykerman RF, Thompson J, Waldie KE, Murphy R, Wall C, Mitchell EA. Antibiotics in the first year of life and subsequent neurocognitive outcomes. Acta Paediatr (2017) 106:87-94. doi:10.1111/apa.13613

62. Wachs TD, Georgieff M, Cusick S, McEwen BS. Issues in the timing of integrated early interventions: contributions from nutrition, neuroscience, and psychological research. Ann N Y Acad Sci (2014) 1308:89-106. doi:10.1111/ nyas. 12314

63. Richardson SS, Daniels CR, Gillman MW, Golden J, Kukla R, Kuzawa C, et al. Society: don't blame the mothers. Nature (2014) 512:131-2. doi:10.1038/ 512131a

64. Yatsunenko T, Rey FE, Manary MJ, Trehan I, Dominguez-Bello MG, Contreras M, et al. Human gut microbiome viewed across age and geography. Nature (2012) 486:222-7. doi:10.1038/nature11053

65. Cheng J, Ringel-Kulka T, Heikamp-de Jong I, Ringel Y, Carroll I, de Vos WM, et al. Discordant temporal development of bacterial phyla and the emergence of core in the fecal microbiota of young children. ISME J (2016) 10:1002-14. doi:10.1038/ismej.2015.177

66. Lozupone CA, Stombaugh JI, Gordon JI, Jansson JK, Knight R. Diversity, stability and resilience of the human gut microbiota. Nature (2012) 489: 220-30. doi:10.1038/nature11550

67. Qin J, Li R, Raes J, Arumugam M, Burgdorf KS, Manichanh C, et al. A human gut microbial gene catalogue established by metagenomic sequencing. Nature (2010) 464:59-65. doi:10.1038/nature08821

68. Rajilić-Stojanović M, de Vos WM. The first 1000 cultured species of the human gastrointestinal microbiota. FEMS Microbiol Rev (2014) 38:996-1047. doi:10.1111/1574-6976.12075

69. Lloyd-Price J, Abu-Ali G, Huttenhower C. The healthy human microbiome. Genome Med (2016) 8:51. doi:10.1186/s13073-016-0307-y

70. Human Microbiome Project Consortium, Gevers D, Knight R, Abubucker S, Badger JH, Chinwalla AT, et al. Structure, function and diversity of the healthy human microbiome. Nature (2012) 486:207-14. doi:10.1038/nature11234

71. Shreiner AB, Kao JY, Young VB. The gut microbiome in health and in disease. Curr Opin Gastroenterol (2015) 31:69-75. doi:10.1097/ MOG.0000000000000139

72. Moya A, Ferrer M. Functional redundancy-induced stability of gut microbiota subjected to disturbance. Trends Microbiol (2016) 24:402-13. doi:10.1016/j. tim.2016.02.002

73. Goodrich JK, Davenport ER, Waters JL, Clark AG, Ley RE. Cross-species comparisons of host genetic associations with the microbiome. Science (2016) 352:532-5. doi:10.1126/science.aad9379

74. Singh RK, Chang H-W, Yan D, Lee KM, Ucmak D, Wong K, et al. Influence of diet on the gut microbiome and implications for human health. J Transl Med (2017) 15:73. doi:10.1186/s12967-017-1175-y

75. Kang SS, Jeraldo PR, Kurti A, Miller MEB, Cook MD, Whitlock K, et al. Diet and exercise orthogonally alter the gut microbiome and reveal independent associations with anxiety and cognition. Mol Neurodegener (2014) 9:36. doi:10.1186/1750-1326-9-36

76. Wilson ID, Nicholson JK. Gut microbiome interactions with drug metabolism, efficacy, and toxicity. Transl Res (2017) 179:204-22. doi:10.1016/j. trsl.2016.08.002 
77. Spanogiannopoulos P, Bess EN, Carmody RN, Turnbaugh PJ. The microbial pharmacists within us: a metagenomic view of xenobiotic metabolism. Nat Rev Microbiol (2016) 14:273-87. doi:10.1038/nrmicro. 2016.17

78. Roy S, Trinchieri G. Microbiota: a key orchestrator of cancer therapy. Nat Rev Cancer (2017) 17:271-85. doi:10.1038/nrc.2017.13

79. Clarke SF, Murphy EF, O’Sullivan O, Lucey AJ, Humphreys M, Hogan A, et al. Exercise and associated dietary extremes impact on gut microbial diversity. Gut (2014) 63:1913-20. doi:10.1136/gutjnl-2013-306541

80. Clark A, Mach N. The crosstalk between the gut microbiota and mitochondria during exercise. Front Physiol (2017) 8:319. doi:10.3389/fphys.2017. 00319

81. Thaiss CA, Levy M, Korem T, Dohnalová L, Shapiro H, Jaitin DA, et al. Microbiota diurnal rhythmicity programs host transcriptome oscillations. Cell (2016) 167:1495-510.e12. doi:10.1016/j.cell.2016.11.003

82. Thaiss CA, Zeevi D, Levy M, Zilberman-Schapira G, Suez J, Tengeler AC, et al. Transkingdom control of microbiota diurnal oscillations promotes metabolic homeostasis. Cell (2014) 159:514-29. doi:10.1016/j.cell.2014. 09.048

83. Bercik P, Denou E, Collins J, Jackson W, Lu J, Jury J, et al. The intestinal microbiota affect central levels of brain-derived neurotropic factor and behavior in mice. Gastroenterology (2011) 141(599-609):609-603. doi:10.1053/j. gastro.2011.04.052

84. Cryan JF. Stress and the microbiota-gut-brain axis: an evolving concept in psychiatry. Can J Psychiatry (2016) 61:201-3. doi:10.1177/0706743716635538

85. Möhle L, Mattei D, Heimesaat MM, Bereswill S, Fischer A, Alutis M, et al. Ly6C(hi) monocytes provide a link between antibiotic-induced changes in gut microbiota and adult hippocampal neurogenesis. Cell Rep (2016) 15:1945-56. doi:10.1016/j.celrep.2016.04.074

86. Prinz M, Erny D, Hagemeyer N. Ontogeny and homeostasis of CNS myeloid cells. Nat Immunol (2017) 18:385-92. doi:10.1038/ni.3703

87. Soenen S, Rayner CK, Jones KL, Horowitz M. The ageing gastrointestinal tract. Curr Opin Clin Nutr Metab Care (2016) 19:12-8. doi:10.1097/ MCO.0000000000000238

88. Salazar N, Arboleya S, Valdés L, Stanton C, Ross P, Ruiz L, et al. The human intestinal microbiome at extreme ages of life. Dietary intervention as a way to counteract alterations. Front Genet (2014) 5:406. doi:10.3389/ fgene.2014.00406

89. Claesson MJ, Cusack S, O'Sullivan O, Greene-Diniz R, de Weerd H, Flannery E, et al. Composition, variability, and temporal stability of the intestinal microbiota of the elderly. Proc Natl Acad Sci U S A (2011) 108(Suppl 1):4586-91. doi:10.1073/pnas.1000097107

90. Sapolsky RM, Krey LC, McEwen BS. The neuroendocrinology of stress and aging: the glucocorticoid cascade hypothesis. Endocr Rev (1986) 7:284-301. doi:10.1210/edrv-7-3-284

91. Prenderville JA, Kennedy PJ, Dinan TG, Cryan JF. Adding fuel to the fire: the impact of stress on the ageing brain. Trends Neurosci (2015) 38:13-25. doi:10.1016/j.tins.2014.11.001

92. Lupien SJ, Schwartz G, Ng YK, Fiocco A, Wan N, Pruessner JC, et al. The Douglas Hospital Longitudinal Study of normal and pathological aging: summary of findings. J Psychiatry Neurosci (2005) 30:328-34. doi:10.record/200604022-004

93. Belvederi Murri M, Pariante C, Mondelli V, Masotti M, Atti AR, Mellacqua Z, et al. HPA axis and aging in depression: systematic review and metaanalysis. Psychoneuroendocrinology (2014) 41:46-62. doi:10.1016/j.psyneuen. 2013.12.004

94. Hood S, Amir S. The aging clock: circadian rhythms and later life.J Clin Invest (2017) 127:437-46. doi:10.1172/JCI90328

95. Liang X, FitzGerald GA. Timing the microbes: the circadian rhythm of the gut microbiome. JBiol Rhythms (2017) 32:505-15. doi:10.1177/ 0748730417729066

96. Erickson KI, Prakash RS, Voss MW, Chaddock L, Heo S, McLaren M, et al. Brain-derived neurotrophic factor is associated with age-related decline in hippocampal volume. J Neurosci (2010) 30:5368-75. doi:10.1523/ JNEUROSCI.6251-09.2010

97. Mattson MP, Maudsley S, Martin B. BDNF and 5-HT: a dynamic duo in age-related neuronal plasticity and neurodegenerative disorders. Trends Neurosci (2004) 27:589-94. doi:10.1016/j.tins.2004.08.001
98. Li S-C, Lindenberger U, Bäckman L. Dopaminergic modulation of cognition across the life span. Neurosci Biobehav Rev (2010) 34:625-30. doi:10.1016/j. neubiorev.2010.02.003

99. Montagne A, Barnes SR, Sweeney MD, Halliday MR, Sagare AP, Zhao Z, et al. Blood-brain barrier breakdown in the aging human hippocampus. Neuron (2015) 85:296-302. doi:10.1016/j.neuron.2014.12.032

100. Deleidi M, Jäggle M, Rubino G. Immune aging, dysmetabolism, and inflammation in neurological diseases. Front Neurosci (2015) 9:172. doi:10.3389/ fnins.2015.00172

101. Franceschi C, Campisi J. Chronic inflammation (inflammaging) and its potential contribution to age-associated diseases. J Gerontol A Biol Sci Med Sci (2014) 69(Suppl 1):S4-9. doi:10.1093/gerona/glu057

102. Fransen F, van Beek AA, Borghuis T, Aidy SE, Hugenholtz F, van der Gaast-de Jongh C, et al. Aged gut microbiota contributes to systemical inflammaging after transfer to germ-free mice. Front Immunol (2017) 8:1385. doi:10.3389/ fimmu.2017.01385

103. Jackson MA, Jackson M, Jeffery IB, Beaumont M, Bell JT, Clark AG, et al. Signatures of early frailty in the gut microbiota. Genome Med (2016) 8:8. doi:10.1186/s13073-016-0262-7

104. Langille MG, Meehan CJ, Koenig JE, Dhanani AS, Rose RA, Howlett SE, et al. Microbial shifts in the aging mouse gut. Microbiome (2014) 2:50. doi:10.1186/s40168-014-0050-9

105. Biagi E, Franceschi C, Rampelli S, Severgnini M, Ostan R, Turroni S, et al. Gut microbiota and extreme longevity. Curr Biol (2016) 26:1480-5. doi:10.1016/j. cub.2016.04.016

106. Scott KA, Ida M, Peterson VL, Prenderville JA, Moloney GM, Izumo T, et al. Revisiting Metchnikoff: age-related alterations in microbiota-gut-brain axis in the mouse. Brain Behav Immun (2017) 65:20-32. doi:10.1016/j. bbi.2017.02.004

107. Masters CL, Bateman R, Blennow K, Rowe CC, Sperling RA, Cummings JL. Alzheimer's disease. Nat Rev Dis Prim (2015) 1:15056. doi:10.1038/nrdp. 2015.56

108. Vogt NM, Kerby RL, Dill-McFarland KA, Harding SJ, Merluzzi AP, Johnson SC, et al. Gut microbiome alterations in Alzheimer's disease. Sci Rep (2017) 7:13537. doi:10.1038/s41598-017-13601-y

109. Cattaneo A, Cattane N, Galluzzi S, Provasi S, Lopizzo N, Festari C, et al. Association of brain amyloidosis with pro-inflammatory gut bacterial taxa and peripheral inflammation markers in cognitively impaired elderly. Neurobiol Aging (2017) 49:60-8. doi:10.1016/j.neurobiolaging.2016. 08.019

110. Harach T, Marungruang N, Duthilleul N, Cheatham V, Mc Coy KD, Frisoni G, et al. Reduction of Abeta amyloid pathology in APPPS1 transgenic mice in the absence of gut microbiota. Sci Rep (2017) 7:41802. doi:10.1038/ srep41802

111. Everard A, Belzer C, Geurts L, Ouwerkerk JP, Druart C, Bindels LB, et al. Cross-talk between Akkermansia muciniphila and intestinal epithelium controls diet-induced obesity. Proc Natl Acad Sci U S A (2013) 110:9066-71. doi:10.1073/pnas.1219451110

112. Minter MR, Zhang C, Leone V, Ringus DL, Zhang X, Oyler-Castrillo P, et al. Antibiotic-induced perturbations in gut microbial diversity influences neuro-inflammation and amyloidosis in a murine model of Alzheimer's disease. Sci Rep (2016) 6:30028. doi:10.1038/srep30028

113. Minter MR, Hinterleitner R, Meisel M, Zhang C, Leone V, Zhang X, et al. Antibiotic-induced perturbations in microbial diversity during post-natal development alters amyloid pathology in an aged APPSWE/PS1 $1 \mathrm{E} 9$ murine model of Alzheimer's disease. Sci Rep (2017) 7:10411. doi:10.1038/ s41598-017-11047-w

114. Brandscheid C, Schuck F, Reinhardt S, Schäfer K-H, Pietrzik CU, Grimm M, et al. Altered gut microbiome composition and tryptic activity of the $5 x F A D$ Alzheimer's mouse model. J Alzheimers Dis (2017) 56:775-88. doi:10.3233/ JAD-160926

115. Kell DB, Pretorius E. On the translocation of bacteria and their lipopolysaccharides between blood and peripheral locations in chronic, inflammatory diseases: the central roles of LPS and LPS-induced cell death. Integr Biol (Camb) (2015) 7:1339-77. doi:10.1039/c5ib00158g

116. Asti A, Gioglio L. Can a bacterial endotoxin be a key factor in the kinetics of amyloid fibril formation? J Alzheimers Dis (2014) 39:169-79. doi:10.3233/ JAD-131394 
117. Sheng JG, Bora SH, Xu G, Borchelt DR, Price DL, Koliatsos VE. Lipopolysaccharide-induced-neuroinflammation increases intracellular accumulation of amyloid precursor protein and amyloid beta peptide in APPswe transgenic mice. Neurobiol Dis (2003) 14:133-45. doi:10.1016/S0969-9961 (03)00069-X

118. Kahn MS, Kranjac D, Alonzo CA, Haase JH, Cedillos RO, McLinden KA, et al. Prolonged elevation in hippocampal $A \beta$ and cognitive deficits following repeated endotoxin exposure in the mouse. Behav Brain Res (2012) 229: 176-84. doi:10.1016/j.bbr.2012.01.010

119. Kitazawa M, Oddo S, Yamasaki TR, Green KN, LaFerla FM. Lipopolysaccharide-induced inflammation exacerbates tau pathology by a cyclin-dependent kinase 5-mediated pathway in a transgenic model of Alzheimer's disease. J Neurosci (2005) 25:8843-53. doi:10.1523/JNEUROSCI. 2868-05.2005

120. Zhan X, Stamova B, Jin L-W, DeCarli C, Phinney B, Sharp FR. Gram-negative bacterial molecules associate with Alzheimer disease pathology. Neurology (2016) 87:2324-32. doi:10.1212/WNL.0000000000003391

121. Bonfili L, Cecarini V, Berardi S, Scarpona S, Suchodolski JS, Nasuti C, et al. Microbiota modulation counteracts Alzheimer's disease progression influencing neuronal proteolysis and gut hormones plasma levels. Sci Rep (2017) 7:2426. doi:10.1038/s41598-017-02587-2

122. Distrutti E, O’Reilly J-A, McDonald C, Cipriani S, Renga B, Lynch MA, et al. Modulation of intestinal microbiota by the probiotic VSL\#3 resets brain gene expression and ameliorates the age-related deficit in LTP. PLoS One (2014) 9:e106503. doi:10.1371/journal.pone.0106503

123. Wang D, Ho L, Faith J, Ono K, Janle EM, Lachcik PJ, et al. Role of intestinal microbiota in the generation of polyphenol-derived phenolic acid mediated attenuation of Alzheimer's disease $\beta$-amyloid oligomerization. Mol Nutr Food Res (2015) 59:1025-40. doi:10.1002/mnfr.201400544

124. Pringsheim T, Jette N, Frolkis A, Steeves TDL. The prevalence of Parkinson's disease: a systematic review and meta-analysis. Mov Disord (2014) 29: 1583-90. doi:10.1002/mds. 25945

125. Hirsch L, Jette N, Frolkis A, Steeves T, Pringsheim T. The incidence of Parkinson's disease: a systematic review and meta-analysis. Neuroepidemiology (2016) 46:292-300. doi:10.1159/000445751

126. Poewe W, Seppi K, Tanner CM, Halliday GM, Brundin P, Volkmann J, et al. Parkinson disease. Nat Rev Dis Prim (2017) 3:17013. doi:10.1038/ nrdp. 2017.13

127. Fasano A, Visanji NP, Liu LWC, Lang AE, Pfeiffer RF. Gastrointestinal dysfunction in Parkinson's disease. Lancet Neurol (2015) 14:625-39. doi:10.1016/ S1474-4422(15)00007-1

128. Hilton D, Stephens M, Kirk L, Edwards P, Potter R, Zajicek J, et al. Accumulation of $\alpha$-synuclein in the bowel of patients in the pre-clinical phase of Parkinson's disease. Acta Neuropathol (2014) 127:235-41. doi:10.1007/ s00401-013-1214-6

129. Shannon KM, Keshavarzian A, Mutlu E, Dodiya HB, Daian D, Jaglin JA, et al. Alpha-synuclein in colonic submucosa in early untreated Parkinson's disease. Mov Disord (2012) 27:709-15. doi:10.1002/mds.23838

130. Holmqvist S, Chutna O, Bousset L, Aldrin-Kirk P, Li W, Björklund T, et al. Direct evidence of Parkinson pathology spread from the gastrointestinal tract to the brain in rats. Acta Neuropathol (2014) 128:805-20. doi:10.1007/ s00401-014-1343-6

131. Svensson E, Horváth-Puhó E, Thomsen RW, Djurhuus JC, Pedersen L, Borghammer P, et al. Vagotomy and subsequent risk of Parkinson's disease. Ann Neurol (2015) 78:522-9. doi:10.1002/ana.24448

132. Hasegawa S, Goto S, Tsuji H, Okuno T, Asahara T, Nomoto K, et al. Intestinal dysbiosis and lowered serum lipopolysaccharide-binding protein in Parkinson's disease. PLoS One (2015) 10:e0142164. doi:10.1371/journal. pone. 0142164

133. Keshavarzian A, Green SJ, Engen PA, Voigt RM, Naqib A, Forsyth CB, et al. Colonic bacterial composition in Parkinson's disease. Mov Disord (2015) 30:1351-60. doi:10.1002/mds.26307

134. Minato T, Maeda T, Fujisawa Y, Tsuji H, Nomoto K, Ohno K, et al. Progression of Parkinson's disease is associated with gut dysbiosis: two-year follow-up study. PLoS One (2017) 12:e0187307. doi:10.1371/journal.pone. 0187307

135. Unger MM, Spiegel J, Dillmann K-U, Grundmann D, Philippeit H, Bürmann J, et al. Short chain fatty acids and gut microbiota differ between patients with Parkinson's disease and age-matched controls. Parkinsonism Relat Disord (2016) 32:66-72. doi:10.1016/j.parkreldis.2016.08.019

136. Bedarf JR, Hildebrand F, Coelho LP, Sunagawa S, Bahram M, Goeser F, et al. Functional implications of microbial and viral gut metagenome changes in early stage L-DOPA-naïve Parkinson's disease patients. Genome Med (2017) 9:39. doi:10.1186/s13073-017-0428-y

137. Fasano A, Bove F, Gabrielli M, Petracca M, Zocco MA, Ragazzoni E, et al. The role of small intestinal bacterial overgrowth in Parkinson's disease. Mov Disord (2013) 28:1241-9. doi:10.1002/mds.25522

138. Huang H-K, Wang J-H, Lei W-Y, Chen C-L, Chang C-Y, Liou L-S. Helicobacter pylori infection is associated with an increased risk of Parkinson's disease: a population-based retrospective cohort study. Parkinsonism Relat Disord (2017) 47:26-31. doi:10.1016/j.parkreldis.2017.11.331

139. Doulberis M, Kotronis G, Thomann R, Polyzos SA, Boziki M, Gialamprinou D, et al. Review: impact of Helicobacter pylori on Alzheimer's disease: what do we know so far? Helicobacter (2018) 23:e12454. doi:10.1111/hel.12454

140. Sampson TR, Debelius JW, Thron T, Janssen S, Shastri GG, Ilhan ZE, et al. Gut microbiota regulate motor deficits and neuroinflammation in a model of Parkinson's disease. Cell (2016) 167:1469-80.e12. doi:10.1016/j. cell.2016.11.018

141. Friedland RP. Mechanisms of molecular mimicry involving the microbiota in neurodegeneration. J Alzheimers Dis (2015) 45:349-62. doi:10.3233/ JAD-142841

142. Hill JM, Lukiw WJ. Microbial-generated amyloids and Alzheimer's disease (AD). Front Aging Neurosci (2015) 7:9. doi:10.3389/fnagi.2015.00009

143. Ransohoff RM. How neuroinflammation contributes to neurodegeneration. Science (2016) 353:777-83. doi:10.1126/science.aag2590

144. Kumar DKV, Choi SH, Washicosky KJ, Eimer WA, Tucker S, Ghofrani J, et al. Amyloid- $\beta$ peptide protects against microbial infection in mouse and worm models of Alzheimer's disease. Sci Transl Med (2016) 8:340ra72. doi:10.1126/ scitranslmed.aaf1059

145. Burgos R, Bretón I, Cereda E, Desport JC, Dziewas R, Genton L, et al. ESPEN guideline clinical nutrition in neurology. Clin Nutr (2017) 37:354-96. doi:10.1016/j.clnu.2017.09.003

146. Mackowiak PA. Recycling Metchnikoff: probiotics, the intestinal microbiome and the quest for long life. Front public Heal (2013) 1:52. doi:10.3389/ fpubh.2013.00052

147. Podolsky SH. Metchnikoff and the microbiome. Lancet (London, England) (2012) 380:1810-1. doi:10.1016/S0140-6736(12)62018-2

148. Blaser MJ, Kirschner D. The equilibria that allow bacterial persistence in human hosts. Nature (2007) 449:843-9. doi:10.1038/nature06198

149. Kuntz TM, Gilbert JA. Introducing the microbiome into precision medicine. Trends Pharmacol Sci (2017) 38:81-91. doi:10.1016/j.tips.2016.10.001

150. Mancuso C, Santangelo R. Alzheimer's disease and gut microbiota modifications: the long way between preclinical studies and clinical evidence. Pharmacol Res (2017). doi:10.1016/j.phrs.2017.12.009

151. Hanage WP. Microbiology: microbiome science needs a healthy dose of scepticism. Nature (2014) 512:247-8. doi:10.1038/512247a

Conflict of Interest Statement: The authors declare that the research was conducted in the absence of any commercial or financial relationships that could be construed as a potential conflict of interest.

Copyright (C) 2018 Calvani, Picca, Lo Monaco, Landi, Bernabei and Marzetti. This is an open-access article distributed under the terms of the Creative Commons Attribution License (CC BY). The use, distribution or reproduction in other forums is permitted, provided the original author(s) and the copyright owner are credited and that the original publication in this journal is cited, in accordance with accepted academic practice. No use, distribution or reproduction is permitted which does not comply with these terms. 